\title{
Adherence to the Mediterranean diet in Portuguese university students
}

\author{
Adesão à Dieta Mediterrânea em estudantes universitários Portugueses \\ Cíntia Ferreira-Pêgo ${ }^{1,2}$, Joana Rodrigues ${ }^{2}$, Adriana Costa ${ }^{2}$, Bruno Sousa $^{1,2}$ \\ ${ }^{1}$ CBIOS Lusófona's Research Center for Biosciences and Health Technologies, Av. Campo Grande 376, 1749-024 Lisbon, \\ Portugal. \\ ${ }^{2}$ School of Sciences and Health Technologies, Universidade Lusófona de Humanidades e Tecnologias, Lisboa, Portugal \\ e-mail: bruno.sousa@mail.com
}

\begin{abstract}
Mediterranean diet (MedDiet) is one of the most recognized healthy dietary patterns. Despite the beneficial effects of healthy eating habits on academic performance, university students make unhealthy food choices. Crosssectional information regarding MedDiet adherence was collected in 305 students from the Universidade Lusófona de Humanidades e Tecnologias, from different academic courses, related or not with health sciences. To assess the MedDiet adherence, the participants completed a validated 14-point questionnaire. Approximately $29 \%$ of the total population presented poor ( $<5$ points) MedDiet adherence, meanwhile $59 \%$ presented an average (between 6 and 9 points) adherence and only $12.50 \%$ presented a high ( $>10$ points) MedDiet adherence.

Nutrition students presented the highest MedDiet adherence of all the students analyzed. Pharmaceutical students, although being health professionals, showed poor adherence to the MedDiet, similar to students from courses not related to health sciences.
\end{abstract}

Keywords: Mediterranean Diet; Mediterranean Diet adherence; health sciences students; university students; Portuguese students

\section{Resumo}

A dieta mediterrânica (DM) é um dos padrões alimentares saudáveis mais reconhecidos. Apesar dos efeitos benéficos dos hábitos alimentares saudáveis no desempenho académico, os estudantes universitários apresentam escolhas alimentares pouco saudáveis. Foi feita uma análise observacional transversal sobre a adesão à DM em 305 estudantes da Universidade Lusófona de Humanidades e Tecnologias, de diferentes cursos académicos, relacionados ou não com as ciências da saúde. Para avaliação da adesão à DM, os participantes responderam a um questionário validado de 14 pontos. Aproximadamente, $29 \%$ do total da população apresentou fraca $(<5$ pontos $)$ adesão à DM, enquanto 59\% apresentaram uma adesão média (entre 6 e 9 pontos) e apenas 12,50\% apresentaram uma alta ( $>10$ pontos) adesão à $\mathrm{DM}$. Os alunos de ciências farmacêuticas e os outros estudantes não relacionados com as ciências da saúde apresentaram um risco significativamente maior de baixa adesão à DM.

Os estudantes de nutrição apresentaram a maior adesão à DM, em comparação com todos os alunos analisados. Estudantes de ciências farmacêuticas, apesar de serem profissionais de saúde, apresentaram baixa adesão à DM, semelhante aos alunos de cursos não relacionados com ciências da saúde.

Palavras-chave: Dieta Mediterrânea; Adesão à dieta mediterrânica; estudantes de ciências da saúde; Estudantes universitários; Estudantes portugueses 


\section{Introduction}

One of the most recognized healthy dietary patterns is the Mediterranean diet (MedDiet), which has been associated with several health benefits, including reducing the risk of type 2 diabetes (1-3), metabolic syndrome $(4,5)$, and major cardiovascular events $(6)$. This dietary pattern is characterized by high consumption of vegetables, legumes, grains, fruits, nuts, and extra virgin olive oil, moderate consumption of fish, and low consumption of red and processed meat, pastries, butter and creams $(7,8)$

Although the beneficial effects of a healthy dietary pattern, like the Mediterranean diet, on academic performance are well-known $(9,10)$, the majority of university students worldwide tend to follow rather unhealthy eating habits (11-14). During university studies, some students are transitioning from their parental house to independent adult life, while facing the challenge to make their own food choices for the first time (15). Particularly, students living away from home tend to develop unfavorable eating habits (14), showing a significant change towards deteriorated, globalized behaviors $(16,17)$.

An unhealthy dietary behavior observed during the university years, is followed by increased weight gain (18), which might often be consolidated for life (15-21). It is also known that the timing of becoming overweight is also crucial for the relative risk of survival in both genders (22).

Therefore, given the importance of maintaining a healthy dietary pattern during youth and early adulthood, the main aim of the present study was to evaluate the adherence to the Mediterranean diet among students from several academic courses, related or not to health sciences, from the Universidade Lusófona de Humanidades e Tecnologias in Lisbon, Portugal.

\section{Material and Methods}

\section{Design and study population}

The present study is a cross-sectional analysis supported by a questionnaire designed to assess the adherence to the MedDiet. The study population was university students from nutrition sciences, pharmaceutical sciences, sports and other courses not related to health sciences area. All the participants ( $\geq 18$ years) were purposively selected among students of these courses at Universidade Lusófona de Humanidades e Tecnologias, during the school year of 2017/2018. The questionnaires were given to 2 nd year students of the nutrition sciences de-

\section{Introdução}

Um dos padrões alimentares saudáveis mais reconhecidos é a dieta mediterrânica (DM), que tem sido associada a vários benefícios para a saúde, incluindo a redução do risco de diabetes tipo 2 (1-3), síndrome metabólica $(4,5)$ e eventos cardiovasculares (6). Esse padrão alimentar é caracterizado pelo alto consumo de vegetais, leguminosas, grãos, frutas, nozes e azeite virgem extra, consumo moderado de peixe e baixo consumo de carnes vermelhas e processadas, doces, manteiga e molhos $(7,8)$

Embora sejam conhecidos os efeitos benéficos de um padrão alimentar saudável, como a DM, no desempenho acadêmico $(9,10)$, a maioria dos estudantes universitários de todo o mundo segue hábitos alimentares pouco saudáveis (11-14). Durante os estudos universitários, alguns alunos saem da casa dos pais para começar uma vida adulta independente, enfrentando o desafio de fazer as suas próprias escolhas alimentares pela primeira vez (15). Particularmente, os estudantes que vivem fora de casa tendem a desenvolver hábitos alimentares desfavoráveis (14), mostrando uma mudança significativa em relação aos comportamentos anteriores $(16,17)$.

Um comportamento alimentar pouco saudável, observado durante os anos da universidade, é seguido por um aumento do ganho de peso (18), que pode, muitas vezes, ser consolidado durante toda a vida (15-21). Sabe-se também que o excesso de peso é crucial para o risco relativo de sobrevivência em ambos os sexos (22).

Portanto, dada a importância de manter um padrão alimentar saudável durante a juventude e início da vida adulta, o objetivo principal do presente estudo foi o de avaliar a adesão à DM entre estudantes de diversos cursos académicos, relacionados ou não com as ciências da saúde, da Universidade Lusófona de Humanidades e Tecnologias em Lisboa, Portugal.

\section{Material e Métodos}

\section{Desenho e estudo da população}

O presente estudo é uma análise observacional transversal apoiada por um questionário com o objetivo de avaliar a adesão à DM. A população estudada incluiu estudantes universitários de ciências da nutrição, ciências farmacêuticas, desporto e outros cursos não relacionados à área de ciências da saúde. Os participantes $(\geq 18$ anos) foram selecionados entre os alunos destes cursos na Universidade Lusófona de Humanidades e Tecnologias, no ano letivo 2017/2018. Os questionários foram aplicados pelos alunos do $2^{\circ}$ ano do curso de ciências da 
gree. Each one was responsible for the recruitment of at least 10 other students from the university, during the period of a week, who were then required to answer the questionnaire for adherence to MedDiet individually. The effective sample size for the present study was 305 participants. The anonymity of the participants was maintained at all times, since the questionnaires did not contain any personal information.

\section{Assessment of the Mediterranean diet}

In order to assess the adherence to the MedDiet, the participants were given a validated 14-item questionnaire (23-25). This 14-item validated survey consisted of 12 questions on food consumption frequency and 2 questions on food intake habits considered characteristic of the Mediterranean diet (Do you use olive oil as the principal source of fat for cooking? and Do you prefer to eat chicken, turkey or rabbit instead of beef, pork, hamburgers or sausages?). Each question was scored 0 (if the condition was not met) or 1 (if the condition was met). The final score ranged from 0 to 14 points. Using this score, we have categorized MedDiet adherence into three categories: poor ( $\leq 5$ points), average (between 6 and 9 points) and high adherence ( $\geq 10$ points).

\section{Statistical analysis}

Data are presented either as means and standard deviation (SD) for continuous variables or numbers and percentages for dichotomous variables. We compared the distribution of the selected characteristics between groups using $\chi^{2}$ tests for categorical variables or Student's t-tests or analysis of variance (ANOVA), as appropriate, for continuous variables. The Bonferroni post-hoc test for multiple comparisons was used. Logistical regression analyses were performed to assess the associations between presenting low MedDiet adherence (score $\leq 10$ points over 14) (as dependent variable) and gender, age, or academic degree (as exposure). The models were adjusted for gender, age, and academic course, except when any of these variables were the independent variable. All statistical tests were two-tailed and the significance level was set at $p<0.05$. All analyses were performed using the SPSS software version 22.0 (SPSS Inc, Chicago, IL).

\section{Results}

A total of 305 students from the Universidade Lusófona de Humanidades e Tecnologia (133 men and 172 women) took part in the present analyses with a mean age of 21.47 (SD 3.58) years. The general characteristics of the studied population according to academic courses nutrição. Cada um foi responsável pelo recrutamento de pelo menos 10 outros estudantes da universidade, durante o período de uma semana, para o preenchimento individual do questionário de adesão à DM. O tamanho efetivo da amostra para o presente estudo foi de 305 participantes. $\mathrm{O}$ anonimato dos participantes foi mantido em todos o momento, uma vez que os questionários não continham qualquer informação pessoal.

\section{Avaliação da dieta Mediterrânica}

Para avaliar a adesão à DM, os participantes receberam um questionário validado de 14 itens (23-25). Este questionário é composto por 12 perguntas sobre frequência de consumo alimentar e 2 perguntas sobre hábitos alimentares considerados característicos da DM (Usa azeite como principal fonte de gordura para cozinhar? e Prefere comer frango, peru ou coelho em vez de carne de porco, hambúrgueres ou salsichas?). Cada questão foi pontuada com 0 (se a condição não foi atendida) ou 1 (se a condição foi atendida). A pontuação final variou de 0 a 14 pontos. Usando esta pontuação, classificámos a adesão à DM em 3 categorias: fraca $(\leq 5$ pontos), média (entre 6 e 9 pontos) e alta ( $\geq 10$ pontos).

\section{Análise estatística}

Os dados são apresentados como médias e desvio padrão (DP) para variáveis contínuas ou números e percentagens para variáveis dicotómicas. Comparámos a distribuição das características selecionadas entre os grupos usando testes de Pearson $\chi^{2}$ para variáveis categóricas ou testes $t$ de Student ou análise de variância (ANOVA), conforme apropriado, para variáveis contínuas. Foi usado o teste post-hoc de Bonferroni para comparações múltiplas. Foram efetuadas análises de regressão logística de forma a avaliar as associações entre apresentar baixa adesão à $\mathrm{DM}(\leq 10$ pontos num total de 14) e sexo, idade ou grau académico como variáveis de exposição. Os modelos foram ajustados para sexo, idade e curso académico, exceto quando qualquer dessas variáveis fosse a variável independente. Todos os testes estatísticos foram bicaudais e o nível de significância foi estabelecido em $\mathrm{P}<0,05$. Todas as análises foram realizadas utilizando o software SPSS versão 22.0 (SPSS Inc, Chicago, IL).

\section{Resultados}

Um total de 305 estudantes da Universidade Lusófona de Humanidades e Tecnologia (133 homens e 172 mulheres) participaram nas presentes análises com uma idade média de 21,47 (DP: 3.58) anos. As características gerais da população estudada de acordo com os 
are summarized in Table 1. The Nutrition students presented a significantly higher adherence to the MedDiet compared to those studying pharmaceutical sciences and also other courses not related to health sciences. No differences related to gender and age were observed between the different academic courses. Otherwise, men presented significantly lower [mean 6.46 (SD 2.34)] adherence to the MedDiet compared to women [mean $7.06(\mathrm{SD} 2.23)]$ in all courses $(p=0.022)$ (data not shown). Approximately the $29 \%$ of the total population presented poor MedDiet adherence with 5 or fewer points over the 14 point questionnaire, while $59 \%$ presented an average adherence, and only $12.50 \%$ of the population presented a high adherence to the MedDiet, with a score of 10 or more points. Significantly differences were observed between academic courses. The nutrition sciences degree presented more students in the highest category of MedDiet adherence compared to the rest. The pharmaceutical students presented poor adherence to the MedDiet, similar to those from other courses not related to health sciences. Comparable results were observed when the analyses were performed by gender. These results are presented in Table 2. The odds of presenting adherence to the MedDiet lower than 10 points (Table 3) were significantly higher in men and younger students in the fully adjusted model. Pharmaceutical students and other students from academic courses not related to health sciences, presented a statistically significant higher risk of poor MedDiet adherence compared to nutrition students. However, when other students not related to health sciences were considered as reference, nutrition [OR (odds ratio) 0.14 (95\%CI 0.06-0.37)] and sports [OR 0.33 (95\%CI 0.13$0.80)$ ] students presented significantly lower probability of presenting less than 10 points in adherence to the Mediterranean diet questionnaire (data not shown). Table 4 shows the consumption of different food and food groups according to academic degree. The students from nutrition sciences presented significantly higher consumption of vegetables, fruits, and nuts compared to those students from other university courses. cursos académicos estão resumidas na Tabela 1. Os estudantes de ciências da nutrição apresentaram uma adesão significativamente maior à DM em comparação com aqueles que estudam ciências farmacêuticas e também com os estudantes dos cursos não relacionados às ciências da saúde. Por outro lado, os indivíduos do sexo masculino apresentaram adesão significativamente menor [média: 6,46 (DP: 2,34)] à DM em relação às mulheres [média: 7,06 (DP: 2,23)] em todos os cursos $(p=0,022)$ (dados não mostrados). Aproximadamente $29 \%$ do total da população apresentou fraca adesão à DM com 5 ou menos pontos sobre o questionário de 14 pontos, enquanto $59 \%$ apresentaram uma adesão média e apenas $12,50 \%$ da população apresentou uma alta adesão à DM, com 10 ou mais pontos. Diferenças significativas foram observadas entre os cursos académicos. A licenciatura em ciências da nutrição apresentou mais alunos na categoria mais alta de adesão à DM, em comparação com os restantes. Os estudantes de ciências farmacêuticas apresentaram pouca adesão à DM, semelhante aos de outros cursos não relacionados com as ciências da saúde. Resultados similares foram observados quando as análises foram realizadas por sexo. Estes resultados são apresentados na Tabela 2. As probabilidades de apresentar uma adesão à DM inferior a 10 pontos (Tabela 3 ) foram significativamente mais elevadas nos indivíduos do sexo masculino e estudantes mais jovens no modelo totalmente ajustado. Estudantes de ciências farmacêuticas e estudantes não relacionados às ciências da saúde apresentaram uma associação significativamente maior de baixa adesão à DM quando os estudantes de ciências da nutrição foram considerados como referência. No entanto, quando outros estudantes não relacionados às ciências da saúde foram considerados como referência, os estudantes de nutrição [OR: 0,14 (IC95\%: 0,06-0,37)] e de desporto [OR: 0,33 (IC95\%: 0,13-0,80)] apresentaram significativamente menor probabilidade de apresentar fraca adesão à DM (dados não mostrados). A Tabela 4 mostra o consumo de diferentes alimentos segundo o curso académico. Os estudantes de ciências da nutrição apresentaram um consumo significativamente maior de vegetais, frutas e nozes, em comparação com os estudantes de outros cursos universitários. 
Table 1/ Tabela 1 - General characteristics of the study population categorized by academic course. Age and MedDiet adherence expressed as means (SD) and gender categories expressed as percentages (n). *p values for comparisons between groups were tested by Student's t-test or Pearson $\chi 2$ as appropriate. c Mediterranean diet adherence expressed in points (score: $0-14$ points). Post-Hoc difference between $a$ and $b$ was tested by the Bonferroni test $(\mathrm{p}<0.05)$ / Características gerais da população estudada categorizada por curso. Idade e adesão à DM expressos em média (DP) e categorias de sexo expressos em percentagens (n). *Valores p para comparações entre grupos foram testados mediante teste $t$ de Student ou Pearson $\chi^{2}$ como apropriado. c Adesão à dieta Mediterrânea expressa em pontos (resultado: 0 a 14 pontos). Diferenças PostHoc entre $\mathrm{a} e \mathrm{~b}$ foram testadas através do teste de Bonferroni $(\mathrm{p}<0.05)$.

\begin{tabular}{lcccccc}
\hline & $\begin{array}{c}\text { All population/ } \\
\text { População tot. } \\
(\mathrm{n}=305)\end{array}$ & $\begin{array}{c}\text { Nutrition/ } \\
\text { Nutrição } \\
(\mathrm{n}=30)\end{array}$ & $\begin{array}{c}\text { Pharmacy/ } \\
\text { Farmácia } \\
(\mathrm{n}=38)\end{array}$ & $\begin{array}{c}\text { Sports/ } \\
\text { Desporto } \\
(\mathrm{n}=54)\end{array}$ & $\begin{array}{c}\text { Others/ } \\
\text { Outros } \\
(\mathrm{n}=183)\end{array}$ & $\begin{array}{c}\boldsymbol{p} \text { - } \\
\text { value }^{*}\end{array}$ \\
\hline Men/ Homem & $43.60(133)$ & $6.80(9)$ & $13.50(18)$ & $22.60(30)$ & $57.10(76)$ & \\
Women/ Mulher & $56.4(172)$ & $12.20(21)$ & $11.60(20)$ & $14.00(24)$ & $62.20(107)$ & 0.115 \\
$\begin{array}{l}\text { Age/ idade, } \\
\text { years/ anos }\end{array}$ & $21.47(3.58)$ & $22.93(5.87)$ & $20.76(2.69)$ & $21.68(3.05)$ & $21.32(3.36)$ & 0.071 \\
$\begin{array}{l}\text { MedDiet } \\
\text { adherence } / \\
\text { adesão à DM }\end{array}$ & $6.80(2.30)$ & $8.33(2.45)^{\mathrm{a}}$ & $6.58(2.01)^{\mathrm{b}}$ & $7.31(2.09)$ & $6.44(2.27)^{\mathrm{b}}<0.001$ \\
\hline
\end{tabular}

Table 2/ Tabela 2 - Percentage of poor, average, or high* adherence to the MedDiet according to an academic course. Data expressed as percentages (n). *Adherence to the MedDiet was categorized into poor ( $<5$ points), average (between 6 and 9 points) and high $(>10$ points). ap values for comparisons between groups were tested by Pearson $\chi 2$./ Percentagem de fraca, media e alta adesão à Dieta Mediterrânea de acordo com o curso. Dados expressos como percentagem (n). *Adesão à dieta Mediterrânea foi categorizada como fraca $(<5$ pontos), media (6-9 pontos) ou alta ( $>10$ pontos). aValores $\mathrm{P}$ para comparações entre grupos foram testados mediante teste de Pearson $\chi 2$.

\begin{tabular}{|c|c|c|c|c|c|c|}
\hline$\overline{\text { Points/ Pontos }}$ & $\begin{array}{c}\text { All population/ } \\
\text { População total } \\
(n=305)\end{array}$ & $\begin{array}{c}\text { Nutrition/ } \\
\text { Nutrição } \\
(\mathrm{n}=30)\end{array}$ & $\begin{array}{c}\text { Pharmacy/ } \\
\text { Farmácia } \\
(\mathrm{n}=38)\end{array}$ & $\begin{array}{c}\text { Sports/ } \\
\text { Desporto } \\
(\mathrm{n}=54)\end{array}$ & $\begin{array}{l}\text { Others/ } \\
\text { Outros } \\
(\mathrm{n}=183)\end{array}$ & $p$-value $e^{a}$ \\
\hline $\begin{array}{l}\text { All population/ } \\
\text { População total } \\
\leq 5\end{array}$ & $28.90(88)$ & $10.00(3)$ & $31.60(12)$ & $20.40(11)$ & $33.90(62)$ & \multirow{3}{*}{$<0.001$} \\
\hline $6-9$ & $58.70(179)$ & $50.00(15)$ & $63.20(24)$ & $61.10(33)$ & $58.50(107)$ & \\
\hline$\geq 10$ & $12.50(38)$ & $40.00(12)$ & $5.30(2)$ & $18.50(10)$ & $7.70(14)$ & \\
\hline \multicolumn{7}{|l|}{ Men/ Homem } \\
\hline$\leq 5$ & 33.10 (44) & $0.00(0)$ & $50.00(9)$ & $26.70(8)$ & $35.50(27)$ & \multirow{3}{*}{0.001} \\
\hline 6-9 & $58.60(78)$ & $55.60(5)$ & $50.00(9)$ & $63.30(19)$ & $59.20(45)$ & \\
\hline$\geq 10$ & $8.30(11)$ & $44.40(4)$ & $0.00(0)$ & $10.00(3)$ & $5.30(4)$ & \\
\hline $\begin{array}{l}\text { Women/ } \\
\text { Mulher } \\
\leq 5\end{array}$ & $25.60(44)$ & $14.30(3)$ & $15.00(3)$ & $12.50(3)$ & $32.70(35)$ & \multirow{3}{*}{0.003} \\
\hline 6-9 & $58.70(101)$ & $47.60(10)$ & $75.00(15)$ & $58.30(14)$ & $57.90(62)$ & \\
\hline$\geq 10$ & $15.70(27)$ & $38.10(8)$ & $10.00(2)$ & $29.20(7)$ & $9.30(10)$ & \\
\hline
\end{tabular}


Table 3/Tabela 3 - Association (odds ratio) between presenting MedDiet adherence $<10$ points and gender, age or academic course. Data expressed as ORs (95\% Confidence Interval). a Gender was coded as 1 for men and 0 for women. Logistic regression model adjusted for gender, age and academic degree (except when gender, age or academic degree were the independent variables/ Associação (odds ratio) entre apresentar adesão à dieta Mediterrânea $<10$ pontos e sexo, idade ou curso. Dados expressos em OR (95\% intervalo de confiança). aSexo foi codificado como 1 para homem e 0 para mulher. Modelo de regressão logística ajustada por sexo, idade e curso académico (exceto quando sexo, idade ou curso académico eram as variáveis independentes).

\begin{tabular}{|c|c|c|c|}
\hline $\begin{array}{l}\text { Variables/ } \\
\text { Variáveis }\end{array}$ & $\begin{array}{c}\text { All population/ } \\
\text { população total } \\
(\mathrm{n}=305)\end{array}$ & $\begin{array}{c}\text { Men/ Homem } \\
(n=133)\end{array}$ & $\begin{array}{c}\text { Women/ Mulher } \\
(n=172)\end{array}$ \\
\hline Gender/Sexo ${ }^{a}$ & $2.30(1.03-5.12)$ & - & - \\
\hline Age/ Idade & $0.91(0.83-0.99)$ & $0.92(0.80-1.06)$ & $0.89(0.79-1.01)$ \\
\hline \multicolumn{4}{|l|}{$\begin{array}{l}\text { Academic degree/ } \\
\text { Curso académico }\end{array}$} \\
\hline $\begin{array}{l}\text { Nutrition/ } \\
\text { Nutrição }\end{array}$ & 1 (ref.) & 1 (ref.) & 1 (ref.) \\
\hline $\begin{array}{l}\text { Pharmacy/ } \\
\text { Farmácia }\end{array}$ & $9.31(1.84-47.26)$ & $49.10(0.00-99.99)$ & 4.59 (0.81-26.09) \\
\hline $\begin{array}{l}\text { Sports/ } \\
\text { Desporto }\end{array}$ & $2.28(0.81-6.46)$ & $6.58(1.10-39.31)$ & $1.35(0.38-4.87)$ \\
\hline Others/ Outros & $6.98(2.73-17.83)$ & $13.51(2.55-71.50)$ & $5.22(1.69-16.18)$ \\
\hline
\end{tabular}

Table 4/Tabela 4 - Consumption of different food or food groups according to an academic course. Data expressed as means (SD) ap values for comparisons between groups were tested by Student's t-test. b Servings per day. c Servings per week. Post-Hoc difference between $d$ and e were tested by the Bonferroni test. Abbreviations: EVOO, Extra Virgin Olive Oil; SSB, Sugar-Sweetened Beverages/ Consumo de diferentes alimentos ou grupos de alimentos de acordo com o curso. Dados expressos em media (DP). aValores P para comparações entre grupos foram testados mediante teste $t$ de Student. b Porções por dia. c Porções por semana. Diferenças Post-Hoc entre d e e foram testadas mediante teste de Bonferroni.

\begin{tabular}{|c|c|c|c|c|c|c|}
\hline & $\begin{array}{l}\text { All population/ } \\
\text { População total } \\
(\mathrm{n}=305)\end{array}$ & $\begin{array}{c}\text { Nutrition/ } \\
\text { Nutrição } \\
(n=30)\end{array}$ & $\begin{array}{c}\text { Pharmacy/ } \\
\text { Farmácia } \\
(\mathrm{n}=38)\end{array}$ & $\begin{array}{c}\text { Sports/ } \\
\text { Desporto } \\
(\mathrm{n}=54)\end{array}$ & $\begin{array}{l}\text { Others/ } \\
\text { Outros } \\
(\mathrm{n}=183)\end{array}$ & $\underset{\text { value }^{a}}{p-}$ \\
\hline EVOO, Azeite b & $2.28(1.51)$ & $2.87(1.22)$ & $2.24(1.22)$ & $2.32(1.52)$ & $2.19(1.59)$ & 0.149 \\
\hline Vegetables, Vegetais ${ }^{\text {b }}$ & $1.64(1.08)$ & $1.95(1.46)^{\mathrm{d}}$ & $1.38(1.00)^{\mathrm{e}}$ & $1.93(1.12)$ & $1.56(0.98)$ & 0.022 \\
\hline Fruits, Frutas ${ }^{\text {b }}$ & $2.13(1.35)$ & $3.22(1.46)^{\mathrm{d}}$ & $1.90(1.06)^{\mathrm{e}}$ & $1.98(0.97)^{\mathrm{e}}$ & $2.04(1.40)^{\mathrm{e}}$ & $<0.001$ \\
\hline $\begin{array}{l}\text { Red meat, Carne } \\
\text { Vermelha }\end{array}$ & $1.35(0.99)$ & $1.13(1.14)$ & $1.26(0.83)$ & $1.30(1.06)$ & $1.41(0.98)$ & 0.476 \\
\hline Butter, Manteiga ${ }^{b}$ & $0.92(0.94)$ & $0.50(0.77)^{\mathrm{d}}$ & $1.03(0.79)$ & $0.79(0.82)$ & $1.01(1.01)^{\mathrm{e}}$ & 0.027 \\
\hline SSB, Refrigerantes ${ }^{b}$ & $0.94(1.70)$ & $0.73(1.36)$ & $1.01(0.98)$ & $0.87(2.79)$ & $0.98(1.44)$ & 0.872 \\
\hline Wine, Vinho ${ }^{c}$ & $0.96(2.80)$ & $0.38(0.96)$ & $1.07(2.07)$ & $0.77(1.04)$ & $1.09(3.42)$ & 0.578 \\
\hline $\begin{array}{l}\text { Legumes, } \\
\text { Leguminosas c }\end{array}$ & $3.03(2.75)$ & $2.78(2.72)$ & $2.42(1.98)$ & $3.02(2.07)$ & $3.21(3.05)$ & 0.415 \\
\hline $\begin{array}{l}\text { Fish and seafood, } \\
\text { Peixe e marisco }^{c}\end{array}$ & $2.57(1.69)$ & $2.92(1.81)$ & $3.03(1.76)$ & $2.69(2.17)$ & $2.39(1.47)$ & 0.094 \\
\hline Bakery, Pastelaría ${ }^{c}$ & $2.41(2.40)$ & $1.95(2.17)$ & $1.80(1.29)^{\mathrm{d}}$ & $2.06(1.69)$ & $2.72(2.72)^{\mathrm{e}}$ & 0.050 \\
\hline Nuts, Frutos secos ${ }^{c}$ & $1.59(2.18)$ & $2.47(1.95)^{\mathrm{d}}$ & $0.79(0.93)^{\mathrm{e}}$ & $1.50(1.51)$ & $1.64(2.49)$ & 0.017 \\
\hline $\begin{array}{l}\text { Tomato, garlic and } \\
\text { onion traditional } \\
\text { sauce, Refugado }^{\text {c }}\end{array}$ & $4.71(3.12)$ & $5.25(3.54)^{\mathrm{e}}$ & $6.60(3.49)^{\mathrm{e}}$ & $3.11(2.42)^{\mathrm{d}}$ & $4.70(2.92)^{\mathrm{e}}$ & $<0.001$ \\
\hline
\end{tabular}




\section{Discussion}

The results of the present study show that the majority of the participants have a low adherence to traditional MedDiet. Females, older students, and nutrition students appeared more sensitive to the adoption of the Mediterranean dietary pattern.

Portugal is the most western country in South Europe and geographically is not adjacent to the Mediterranean sea. However, MedDiet is a settled cultural heritage of the Portuguese population, with ancestral influence from the Mediterranean neighbors (26). Several studies have shown that Mediterranean basin populations tended to abandon the traditional diet during the last decades $(27,28)$ and the same occurred in Portugal $(26)$. The major concern is the early abandonment of MedDiet during childhood, adolescence and youth, leading to the replacement of traditional dietary habits with an increasing consumption of red meat, butter, animal fats, added sugars and salty foods (29-32), which increased the risk of obesity and several health problems (33-35). Research on the MedDiet adherence in Portuguese students is scarce. However, similar results regarding the MedDiet adherence in university students, mainly in nutrition and non-nutrition students were observed in Greece (36). The authors described that nutrition education reversely affects food insecurity, while additionally, the beneficial effects of nutrition knowledge on diet quality are well-known $(37,38)$. Thus, it is possible that the increased nutrition knowledge of nutrition students reduced the prevalence of poor MedDiet.

Corresponding to the results observed during the present analyses, different researchers also described that women $(39,40)$ and older individuals $(40-42)$ are more likely to follow a healthy dietary pattern, such as MedDiet.

These results emphasize the importance of nutritional education programs inside the universities to improve the eating choices of their students, either in courses not related with health sciences but also in pharmaceutical sciences students, in that although being future health professionals their knowledge about nutrition is scarce.

\section{Conclusion}

Students from nutrition sciences showed a higher adherence to the MedDiet compared to students from other academic courses. Pharmaceutical students, although being future health professionals, showed poor adher-

\section{Discussão}

Os resultados do presente estudo indicam que a maioria dos estudantes analisados não segue uma DM tradicional, apresentando um padrão alimentar menos saudável. Mulheres, estudantes mais velhos e estudantes de ciências da nutrição pareceram mais sensíveis à adoção do padrão alimentar mediterrâneo. Portugal é o país mais ocidental do sul da Europa e geograficamente não é banhado pelo mar Mediterrâneo. No entanto, a DM é um património cultural adotado pela população portuguesa, com influência ancestral dos vizinhos do Mediterrâneo (25). Vários estudos mostraram que as populações do Mediterrâneo tenderam a abandonar a dieta tradicional nas últimas décadas $(26,27)$ e o mesmo ocorreu em Portugal (25). A principal preocupação é o abandono precoce da DM durante a infância, adolescência e juventude, levando à substituição de hábitos alimentares tradicionais por um consumo crescente de carne vermelha, manteiga, gorduras animais, açúcares adicionados e alimentos salgados (28-31), o que aumenta o risco de obesidade e vários problemas de saúde (32-34). A pesquisa sobre a adesão à DM em estudantes portugueses é escassa. No entanto, resultados semelhantes em relação à adesão à DM em estudantes universitários, principalmente em estudantes de nutrição, foram observados na Grécia (35). Os autores descreveram que a educação nutricional afeta reversamente a insegurança alimentar, enquanto, adicionalmente, os efeitos benéfi$\cos$ da educação nutricional sobre a qualidade da dieta são bem conhecidos (36,37). Assim, é possível que o aumento do conhecimento nutricional dos estudantes de ciências da nutrição tenha reduzido a prevalência de fraca adesão à DM. Correspondendo aos resultados observados durante a presente análise, diferentes investigadores também descreveram que mulheres $(38,39)$ e indivíduos mais velhos (39-41) são mais propensos a seguir um padrão alimentar saudável, como a DM. Estes resultados ressaltam a importância dos programas de educação nutricional dentro das universidades para melhorar as escolhas alimentares de seus alunos, seja em cursos não relacionados às ciências da saúde, mas também em estudantes de ciências farmacêuticas, que apesar de serem futuros profissionais de saúde, o seu conhecimento sobre nutrição é escasso.

\section{Conclusão}

Os estudantes de ciências da nutrição apresentaram uma adesão à DM superior aos estudantes dos outros cursos académicos. Os estudantes de ciências farmacêuticas, apesar de serem futuros profissionais de saúde, apresen- 
ence to the MedDiet, similar to students from courses not related to health sciences.

\section{Aknowledgements}

The data was collected by students from the $2^{\text {nd }}$ year of Nutrition Sciences of the Universidade Lusófona de Humanidades e Tecnologias. The authors acknowledge all the participants.

\section{Conflict of Interest}

C.F.-P., J.R., A.C., B.S. declare that is no financial or personal relationship that represents any potential conflict of interest. taram uma fraca adesão à DM, exibindo resultados similares aos dos alunos de cursos não relacionados com ciências da saúde.

\section{Agradecimentos}

Os dados foram recolhidos pelos alunos do $2^{\circ}$ ano da licenciatura em ciências da nutrição da Universidade Lusófona de Humanidades e Tecnologias. Os autores agradecem a todos os participantes envolvidos.

\section{Conflitos de Interesse}

C.F.-P., J.R., A.C., B.S. declaram que não há relação financeira ou pessoal que represente qualquer potencial conflito de interesse.

\section{References/ Referências}

1. Schwingshackl L, Missbach B, König J, Hoffmann G. Adherence to a Mediterranean diet and risk of diabetes: a systematic review and meta-analysis. Public Health Nutr. 2014 Aug 22;18(7):1-8.

2. Georgoulis M, Kontogianni MD, Yiannakouris N. Mediterranean diet and diabetes: prevention and treatment. Nutrients . 2014 Apr;6(4):1406-23.

3. Salas-Salvadó J, Bulló M, Estruch R, Ros E, Covas M-I, Ibarrola-Jurado N, et al. Prevention of diabetes with Mediterranean diets: a subgroup analysis of a randomized trial. Ann Intern Med. 2014 Jan 7;160(1):1-10.

4. Babio N, Bulló M, Basora J, Martínez-González MA, Fernández-Ballart J, Márquez-Sandoval F, et al. Adherence to the Mediterranean diet and risk of metabolic syndrome and its components. Nutr Metab Cardiovasc Dis. 2009 Oct;19(8):563-70.

5. Salas-Salvadó J, Fernández-Ballart J, Ros E, Martínez-González M-A, Fitó M, Estruch R, et al. Effect of a Mediterranean diet supplemented with nuts on metabolic syndrome status: one-year results of the PREDIMED randomized trial. Arch Intern Med. 2008 Dec 8 [cited 2014 Feb 10];168(22):2449-58. Available from: http://www.ncbi.nlm.nih.gov/pubmed/19064829

6. Estruch R, Ros E, Salas-Salvadó J, Covas M-I, Corella D, Arós F, et al. Primary prevention of cardiovascular disease with a Mediterranean diet. N Engl J Med. 2013 Apr 4;368(14):1279-90.

7. SCHRODER H. Protective mechanisms of the Mediterranean diet in obesity and type 2 diabetes. J Nutr Biochem. 2007 Mar;18(3):149-60.

8. Bach-Faig A, Berry EM, Lairon D, Reguant J, Trichopoulou A, Dernini S, et al. Mediterranean diet pyramid today. Science and cultural updates. Public Health Nutr. 2011 Dec 13;14(12A):2274-84

9. Florence MD, Asbridge M, Veugelers PJ. Diet Quality and Academic Performance. J Sch Health. 2008 Apr;78(4):209-15.

10. Chacón-Cuberos R, Zurita-Ortega F, Martínez-Martínez A, Olmedo-Moreno E, Castro-Sánchez M. Adherence to the Mediterranean Diet Is Related to Healthy Habits, Learning Processes, and Academic Achievement in Adolescents: A Cross-Sectional Study. Nutrients. 2018 Oct 23;10(11):1566.

11. El Ansari W, Stock C, Mikolajczyk RT. Relationships between food consumption and living arrangements among university students in four European countries - a cross-sectional study. Nutr J. 2012 Apr 24;11(1):28.

12. Tanton J, Dodd LJ, Woodfield L, Mabhala M. Eating Behaviours of British University Students: A Cluster Analysis on a Neglected Issue. Adv Prev Med. 2015;2015:1-8.

13. Deliens T, Clarys P, De Bourdeaudhuij I, Deforche B. Determinants of eating behaviour in university students: a qualitative study using focus group discussions. BMC Public Health. 2014 Dec 18;14(1):53.

14. Papadaki A, Hondros G, A. Scott J, Kapsokefalou M. Eating habits of University students living at, or away from home in Greece. Appetite. 2007 Jul;49(1):169-76.

15. Deshpande S, Basil MD, Basil DZ. Factors influencing healthy eating habits among college students: an application of the health belief model. Health Mark Q. 2009 May 8;26(2):145-64.

16. Steptoe A, Wardle J, Cui W, Baban A, Glass K, Tsuda A, et al. An international comparison of tobacco smoking, beliefs and risk awareness in university students from 23 countries. Addiction. 2002 Dec;97(12):1561-71.

17. Chourdakis M, Tzellos T, Papazisis G, Toulis K, Kouvelas D. Eating habits, health attitudes and obesity indices among medical students in northern Greece. Appetite. 2010 Dec];55(3):722-5.

18. Anderson DA, Shapiro JR, Lundgren JD. The freshman year of college as a critical period for weight gain: an initial evaluation. Eat Behav. 2003 Nov;4(4):363-7.

19. Holben DH, Marshall MB. Position of the Academy of Nutrition and Dietetics: Food Insecurity in the United States. J Acad Nutr Diet. 2017 Dec;117(12):1991-2002.

20. Lee JS, Gundersen C, Cook J, Laraia B, Johnson MA. Food Insecurity and Health across the Lifespan. Adv Nutr. 2012 Sep 1;3(5):744-5.

21. Racette SB, Deusinger SS, Strube MJ, Highstein GR, Deusinger RH. Weight Changes, Exercise, and Dietary Patterns During Freshman and Sophomore Years of College. J Am Coll Heal. 2005 May;53(6):245-51.

22. Adams KF, Leitzmann MF, Ballard-Barbash R, Albanes D, Harris TB, Hollenbeck A, et al. Body Mass and Weight Change in Adults in Relation to Mortality Risk. Am J Epidemiol. 2014 Jan 15;179(2):135-44. 
23. Schröder H, Fitó M, Estruch R, Martínez-González MA, Corella D, Salas-Salvadó J, et al. A short screener is valid for assessing Mediterranean diet adherence among older Spanish men and women. J Nutr. 2011 Jun;141(6):1140-5.

24. Martínez-González MMA, García-Arellano A, Toledo E, Salas-Salvadó J, Buil-Cosiales P, Corella D, et al. A 14-item Mediterranean diet assessment tool and obesity indexes among high-risk subjects: the PREDIMED trial. PLoS One. 2012 Jan;7(8):e43134.

25. Afonso L, Moreira T, Oliveira A. Índices de adesão ao padrão alimentar mediterrânico - a base metodológica para estudar a sua relação com a saúde. Factores de Risco. 2014;31:48-55.

26. Pereira-da-Silva L, Pinto E. Low Adherence to Mediterranean Diet in Portugal: Pregnant Women Nutrition in Portugal and its Repercussions. Acta Med Port. 2016 Oct 31;29(10):658.

27. Vilarnau C, Stracker DM, Funtikov A, da Silva R, Estruch R, Bach-Faig A. Worldwide adherence to Mediterranean Diet between 1960 and 2011. Eur J Clin Nutr. 2018 Nov 28;

28. Galli A, Iha K, Halle M, El Bilali H, Grunewald N, Eaton D, et al. Mediterranean countries' food consumption and sourcing patterns:An Ecological Footprint viewpoint. Sci Total Environ. 2017 Feb;578:383-91.

29. Kontogianni MD, Vidra N, Farmaki A-E, Koinaki S, Belogianni K, Sofrona S, et al. Adherence Rates to the Mediterranean Diet Are Low in a Representative Sample of Greek Children and Adolescents. J Nutr. 2008 Oct 1;138(10):1951-6.

30. Aounallah-Skhiri H, Traissac P, El Ati J, Eymard-Duvernay S, Landais E, Achour N, et al. Nutrition transition among adolescents of a south-Mediterranean country: dietary patterns, association with socio-economic factors, overweight and blood pressure. A cross-sectional study in Tunisia. Nutr J. 2011 Apr 24;10(1):38.

31. Grosso G, Marventano S, Buscemi S, Scuderi A, Matalone M, Platania A, et al. Factors Associated with Adherence to the Mediterranean Diet among Adolescents Living in Sicily, Southern Italy. Nutrients. 2013 Dec 4;5(12):4908-23.

32. Naska A, Trichopoulou A. Back to the future: the Mediterranean diet paradigm. Nutr Metab Cardiovasc Dis. 2014 Mar;24(3):216-9.

33. Haidari F, Shirbeigi E, Cheraghpour M, Mohammadshahi M. Association of dietary patterns with body mass index, waist circumference, and blood pressure in an adult population in Ahvaz, Iran. Saudi Med J. 2014 Sep;35(9):967-74.

34. Medina-Remón A, Kirwan R, Lamuela-Raventós RM, Estruch R. Dietary patterns and the risk of obesity, type 2 diabetes mellitus, cardiovascular diseases, asthma, and neurodegenerative diseases. Crit Rev Food Sci Nutr. 2018 Jan 22;58(2):262-96.

35. Raimundo M, Mira F, Cachulo M da L, Barreto P, Ribeiro L, Farinha C, et al. Adherence to a Mediterranean diet, lifestyle and age-related macular degeneration: the Coimbra Eye Study - report 3. Acta Ophthalmol. 2018 Dec;96(8):e926-32.

36. Theodoridis X, Grammatikopoulou MG, Gkiouras K, Papadopoulou SE, Agorastou T, Gkika I, et al. Food insecurity and Mediterranean diet adherence among Greek university students. Nutr Metab Cardiovasc Dis. 2018 May;28(5):477-85.

37. Mello JA, Gans KM, Risica PM, Kirtania U, Strolla LO, Fournier L. How is food insecurity associated with dietary behaviors? An analysis with lowincome, ethnically diverse participants in a nutrition intervention study. J Am Diet Assoc. 2010 Dec;110(12):1906-11.

38. Hale R, Chhabra S, Zipfel A, Holben D, Vaughn L. Food insecurity, nutrition knowledge, and cooking skills are barriers to healthy eating among food pantry users. 2012;

39. Zazpe I, Sánchez-Tainta A, Toledo E, Sánchez-Villegas A, Martínez-González MÁ. Dietary Patterns and Total Mortality in a Mediterranean Cohort: The SUN Project. J Acad Nutr Diet. 2013 Oct 1;114(1):37-47.

40. Sánchez-Villegas A, Delgado-Rodríguez M, Martínez-González MA, De Irala-Estévez J. Gender, age, socio-demographic and lifestyle factors associated with major dietary patterns in the Spanish Project SUN (Seguimiento Universidad de Navarra). Eur J Clin Nutr. 2003 Feb;57(2):285-92.

41. Tur JA, Romaguera D, Pons A. Adherence to the Mediterranean dietary pattern among the population of the Balearic Islands. Br J Nutr. 2004 Sep;92(3):341-6.

42. Sánchez-Villegas A, Martínez JA, De Irala J, Martínez-González MA. Determinants of the adherence to an "a priori” defined Mediterranean dietary pattern. Eur J Nutr. 2002 Dec;41(6):249-57. 